\title{
Long-term Results of Mini Asymmetric Radial Keratotomy for the Treatment of Keratoconus
}

\author{
${ }^{1}$ Marco Abbondanza, ${ }^{2}$ Gabriele Abbondanza, ${ }^{3}$ Valentina De Felice
}

\section{ABSTRACT}

Aim: To present long-term results of mini asymmetric radial keratotomy (MARK) for the treatment of mild to moderate keratoconus, with an average follow-up of 7.3 years and a minimum of 6 years.

Design: Retrospective clinical study.

Materials and methods: The postoperative outcomes of 66 eyes (45 patients) were selected and retrospectively analyzed. Inclusion criteria were progressive stages I and II keratoconus $(\mathrm{Kc})$ and contact lens intolerance, while exclusion criteria were advanced stages of the pathology and chronic or recurrent ocular infections. Tomography- and topography-guided miniincisions were customised and performed sectorally with a calibrated diamond knife and custom-made corneal markers.

Results: After a minimum follow-up of 6 years, mean keratometry improved in $91 \%$ of cases (from $47.25 \mathrm{D}$ to 44.61 D), and mean pachymetry improved in $98 \%$ of cases (from 446 $\mu \mathrm{m}$ to $484 \mu \mathrm{m}$ ), while best spectacle-corrected visual acuity improved for $95 \%$ of patients, from 0.23 LogMAR (5.9/10) to 0.095 LogMAR (7.7/10).

Conclusion: MARK improved both mean keratometry and mean pachymetry, thus halting the progression of keratoconus, while improving visual acuity. The long-term results suggest that MARK should be considered as one of the conservative treatments for moderate to mild progressive keratoconus.

Keywords: Keratoconus, MARK, Mini asymmetric radial keratotomy.

How to cite this article: Abbondanza M, Abbondanza G, De Felice V. Long-term Results of Mini Asymmetric Radial Keratotomy for the Treatment of Keratoconus. Int J Kerat Ect Cor Dis 2018;7(2):105-109.

Source of support: Nil

Conflict of interest: None

${ }^{1}$ Ophthalmologist and Ophthalmic Surgeon, ${ }^{2}$ Researcher, ${ }^{3}$ Orthoptist

${ }^{1}$ Department of Surgery, Abbondanza Eye Centres, Rome and Milan, Latium and Lombardy, Italy

${ }^{2}$ Department of International Research, The University of Sydney, Sydney, NSW, Australia

${ }^{3}$ Department of Orthoptics, Abbondanza Eye Centres, Rome and Milan, Latium, Italy

Corresponding Author: Marco Abbondanza, Ophthalmologist and Ophthalmic Surgeon, Department of Surgery, Abbondanza Eye Centres, Rome and Milan, Latium and Lombardy, Italy, e-mail: info@abbondanza.org

\section{INTRODUCTION}

Keratoconus $(\mathrm{Kc})$ is one the most extensively studied pathologies in ophthalmology. Identified and described by Nottingham in 1854 , it is a bilateral non-inflammatory corneal ectasia, generally manifested in the second decade of life, which results in progressive and asymmetric corneal distortion and thinning, altered refractive powers and reduced vision. ${ }^{1}$ The history of its treatment is equally long, ${ }^{2}$ and it currently provides invasive and conservative treatments.

The former includes penetrating keratoplasty (PK) and deep anterior lamellar keratoplasty (DALK), ${ }^{3}$ while the latter includes corneal cross-linking (CXL), ${ }^{4}$ the gold standard method for Kc treatment, intracorneal ring segments (IcRS), ${ }^{5}$ circular keratotomy $(\mathrm{CKt}),{ }^{6} \mathrm{mini}$ asymmetric radial keratotomy (MARK), ${ }^{7} \mathrm{radial} / \mathrm{mini}-$ radial keratotomy (RK/Mini-RK), ${ }^{8,9}$ and conductive keratoplasty $(\mathrm{CKp}) .{ }^{10}$ Further, there is a more recent trend involving CXL paired with other procedures (CXL Plus), specifically with photorefractive keratectomy (PRK) (Athens Protocol), ${ }^{11}$ Laser in-situ keratomileusis (LASIK) ${ }^{12} \mathrm{ICrS}^{13}{ }^{13} \mathrm{MARK}$ (Rome Protocol) ${ }^{14} \mathrm{CKp}^{15}$ and phakic intraocular lenses (PIoL). ${ }^{16}$

However, despite its simplicity and effectiveness in halting Kc, CXL does not improve vision, apart from a slight degree of corneal regularisation with epithelium removal, ${ }^{17}$ a condition that justifies the rationale behind the other procedures mentioned above.

The present study focuses on mini asymmetric radial keratotomy, developed in 1993 by the author (MA) as a noninvasive alternative to penetrating keratoplasty. Longterm results are analyzed here, with the aim of expanding the literature concerning conservative treatment for Kc.

\section{MATERIALS AND METHODS}

\section{Study Design}

This is a retrospective observational case series of 45 patients (66 eyes) who underwent MARK with longterm follow-up (7.3 years on average, 6 years minimum), performed by the same surgeon (MA) with the same instruments in two different centers. Out of the circa 1,000 eyes that have been treated with MARK in our 
centers, 66 were selected due to their long-term follow-up and completeness of postoperative data. Preoperative demographics also show that the cohort of patients consisted of 31 males and 14 females, and that mean age was 31.8 years.

Clinical selection included patients affected by progressive stages I and II Kc (Amsler-Krumeich classification) and suffering from contact lens intolerance (CLI), while patients with any chronic or recurrent ocular infections were excluded. All patients underwent a complete clinical examination before the intervention, which was conducted in accordance with Declaration of Helsinki, ethics approval was assessed by the director of the two centers (MA), and a written informed consent for both procedures was required as a part of our preoperative routine.

For the purposes of this study, best spectacle-corrected visual acuity (BSCVA), mean pachymetry and mean keratometry (Kavg) were recorded with a Pentacam (Oculus Optikgerate Gmbh, Wetzlar, Germany) preoperatively and at least 1,3 and 6 years following the intervention. All data were collected in standardized study spreadsheets and entered into Excel 2010 (Microsoft Corporation, Redmond, Washington), which also provided basic statistical outcomes, and visual acuity was measured according to a decimal scale and then converted to the logarithm of the minimum angle of resolution (LogMAR). Steady and improved pachymetric and keratometric values have been used as indicators of successful Kc stabilization, while an increase in BSCVA has been used as the standard indicator of improved visual acuity.

\section{Surgical Procedure}

Mini asymmetric radial keratotomy was originally conceived as a hybrid technique, a surgical synthesis of asymmetric radial keratotomy ${ }^{18}$ —already using sectoral incisions but too prone to hyperopic overcorrectionand mini-RK, less invasive but still applying incision on the full corneal angular span. MARK's conservative nomogram is based on Lindstrom's, ${ }^{19}$ modified so that it performs shorter $(1.75-2.25 \mathrm{~mm}$ instead of $3 \mathrm{~mm})$ and asymmetric (sectorial) mini-incisions (Fig. 1).

Further, the differences between MARK incision and standard RK incisions are very significant, especially when treating a degenerative pathology such as Kc, since MARK surgery:

- Uses very short incisions (approximately $2 \mathrm{~mm}$ ) compared to the full length of RK incisions.

- Applies less deep incisions (approximately 80\% of corneal thickness) compared to RK incisions.

- Requires few (3-5) customized mini-incisions that involve only a limited angular span of the

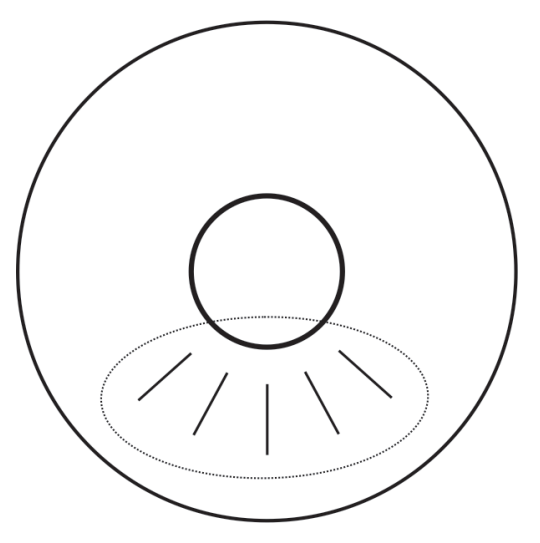

Fig. 1: A diagram of typical MARK mini-incisions, performed only on the corneal sector affected by Kc

cornea, as opposed to the full angular span involved with RK.

- Does not preclude future surgical options such as PK or DALK, due to the fact MARK mini-incisions do not extend beyond a corneal diameter of $8 \mathrm{~mm}$ peripherally, which means that they would be included in the diameter of corneal tissue to be removed and substituted with the corneal graft.

These are remarkably significant differences that are bound to affect long-term stability and safety.

Once the inclusion/exclusion criteria are satisfied, preparation for MARK starts by devising its surgical plan. Since it consists of a small series (3-5) of tomographyand topography-guided centripetal mini-incisions, performed only on the portion of the cornea where Kc is manifest, at circa $80 \%$ of corneal thickness, factors that are carefully considered included the designation of the corneal clear zone, which is to be left as large as possible, along with the determination of the number, position, length, and depth of such mini-incisions. A diamond knife (Meyco, Anton Meyer \& Co. Ltd, Biel, Switzerland) and custom-made double concentric corneal markers (Fig. 2), limiting the incisions to a maximum of $7.5-8 \mathrm{~mm}$ on the external end and a minimum of $3.5-4$

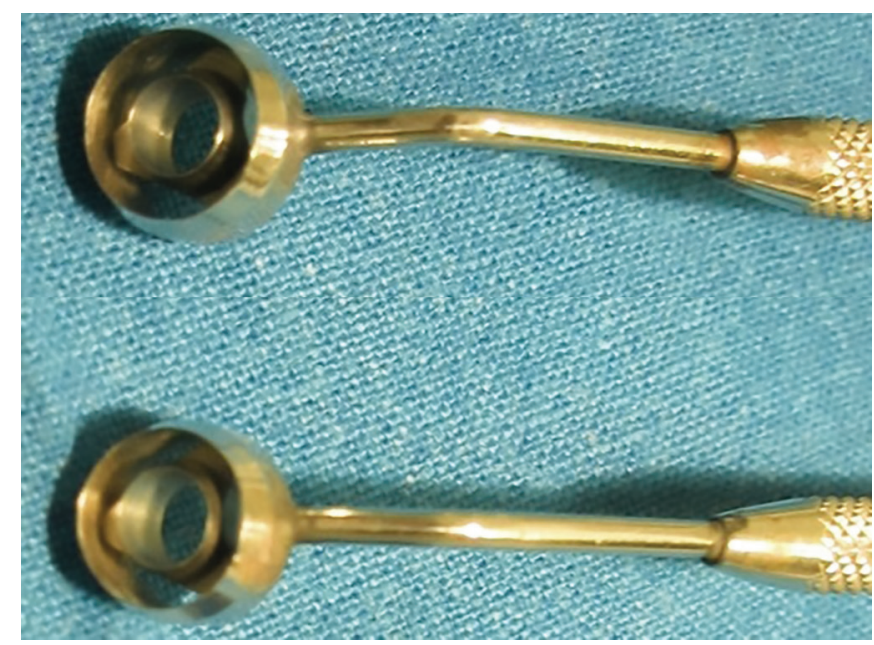

Fig. 2: Abbondanza markers 
$\mathrm{mm}$ on the internal end, are used. Topical anesthesia is applied before the operation (benoxinate chloride $0.4 \%$ ). Following the intervention, a bandage contact lens is placed, and topical antibiotics (levofloxacin hemihydrate $0.5 \%$ ) and nonsteroidal anti-inflammatory drops (diclofenac sodic $0.1 \%$ ) are prescribed.

\section{RESULTS}

\section{Long-term Outcomes}

As mentioned, the key parameters collected and analyzed by this study are best spectacle-corrected visual acuity (BSCVA), mean pachymetry and mean keratometry (Kavg). The former has improved in $95 \%$ of cases (63 out of 66 eyes), from a preoperative average of 0.23 LogMAR (5.9/10) to a postoperative one of $0.095 \operatorname{LogMAR}(7.7 / 10$, Graph 1). The second parameter has improved in $98 \%$ of cases (65 out of 66), from a preoperative average of 446 $\mu \mathrm{m}$ to a postoperative one of $484 \mu \mathrm{m}$ (Graph 2). The third and last parameter employed here, lastly, has improved in $91 \%$ of cases (60 out of 66), from a preoperative average

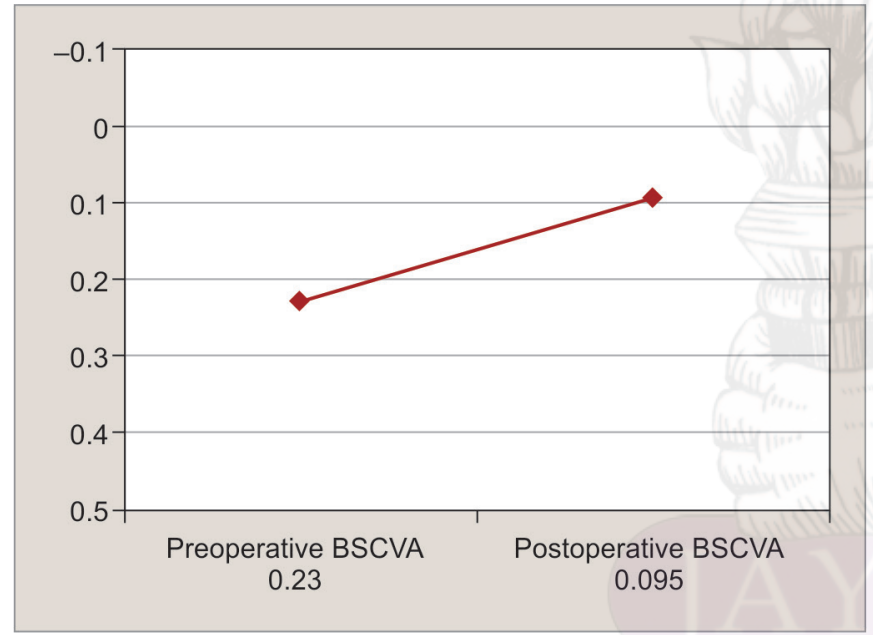

Graph 1: Changes in BSCVA after at least 6 years of follow-up of $47.25 \mathrm{D}$ to a 6-years postoperative average of $44.61 \mathrm{D}$. Moreover, the smaller cohort of patients with a longer follow-up provides further support for the evaluation of MARK's long-term stability. Among those with 9 years of data, average keratometry has improved from 47.13-43 D; among those with 10 years, it has improved from 48.1744.46 D; with 12 years, from 49.23-45.6 D; and, finally, with 13 years of follow-up Kavg has decreased from 48.12-44.72 D (Graph 3). Aggregate data are summarized in Table 1, and the original dataset is available from the corresponding author.

\section{Complications}

No intraoperative or postoperative complications occurred. Corneal microperforations, potentially occurring during the incisional process, did not take place. Some patients experienced minor postoperative discomfort due to a perceived foreign-body sensation, common to a number of corneal procedures, as well as blurriness after the removal of the bandage contact lens, which progressively disappeared.

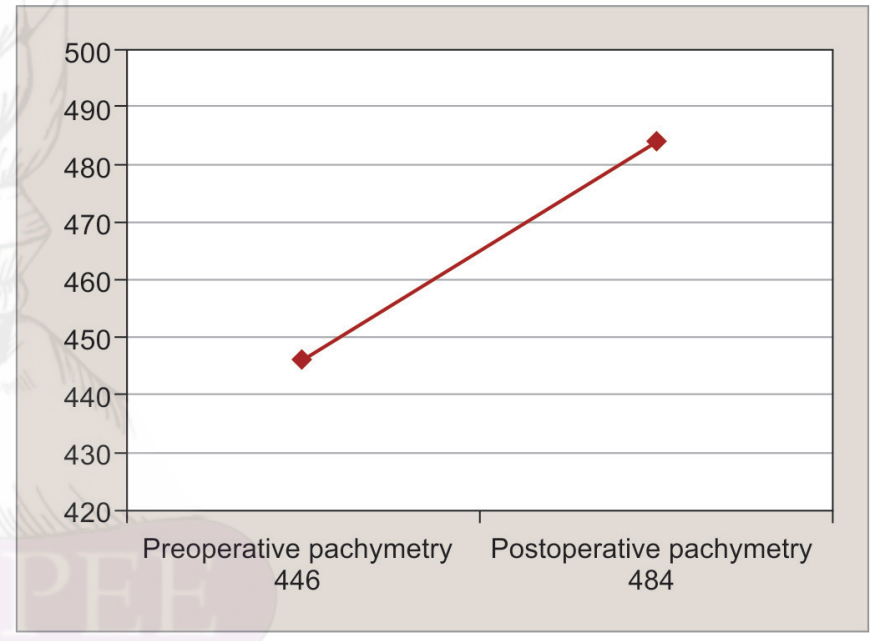

Graph 2: Changes in mean pachymetry after at least 6 years of follow-up

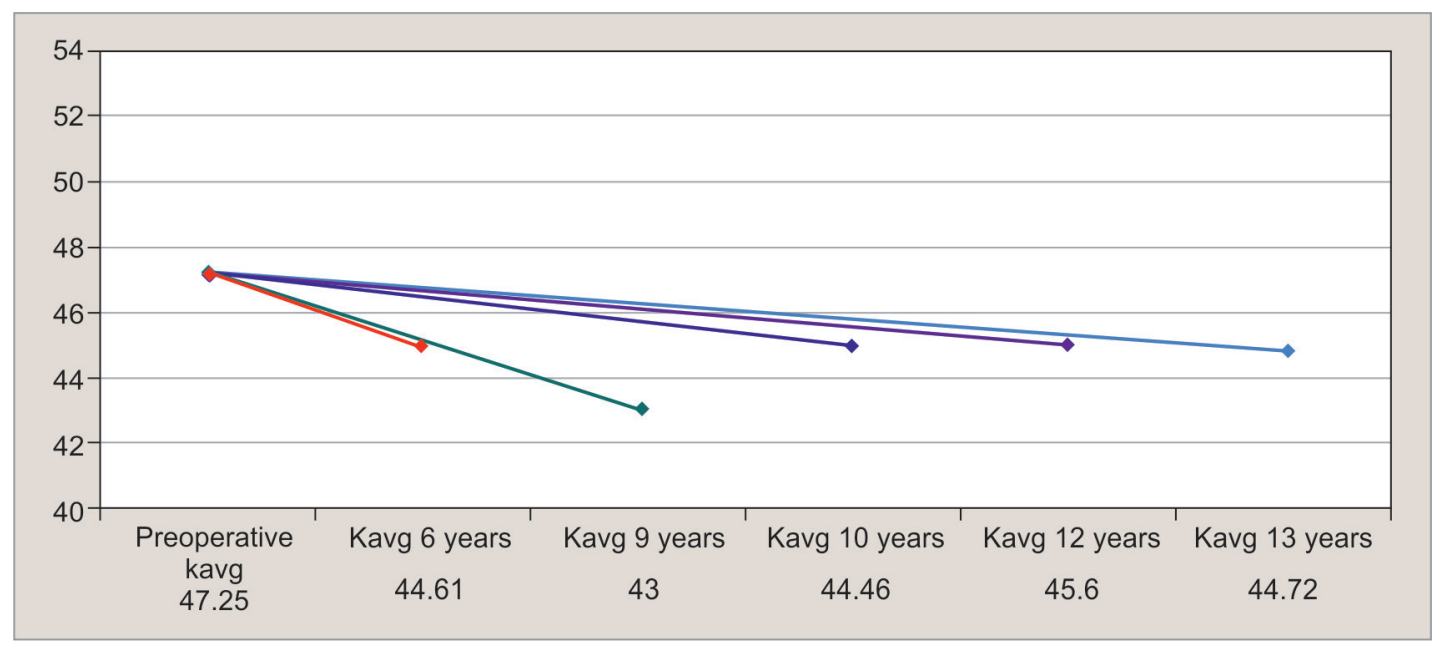

Graph 3: Changes in mean keratometry before and after surgery, with a minimum follow-up of 6 years, common to all eyes, and longer ones, representing smaller cohorts 
Table 1: Summary of long-term outcomes before and after MARK surgery

\begin{tabular}{lccc}
\hline & \multicolumn{3}{c}{ Postoperative } \\
& Preoperative & $(6 \mathrm{y})$ & Difference \\
\hline BSCVA & $\begin{array}{c}0.23 \text { LogMAR } \\
(5.9 / 10)\end{array}$ & $\begin{array}{c}0.095 \\
\text { LogMAR } \\
(7.7 / 10)\end{array}$ & $\begin{array}{c}-0.135 \text { LogMAR } \\
(+1.8 / 10)\end{array}$ \\
& & $484 \mu \mathrm{m}$ & $+38 \mu \mathrm{m}$ \\
Mean & $446 \mu \mathrm{m}$ & & \\
pachymetry & & $44.61 \mathrm{D}$ & $-2.64 \mathrm{D}$ \\
Kavg & $47.25 \mathrm{D}$ & &
\end{tabular}

\section{DISCUSSION}

These data allow for a number of considerations. First, they confirm that MARK is effective in stabilizing keratoconus, since average keratometry and average pachymetry have improved by $91 \%$ and $98 \%$ of cases respectively. Second, MARK provides visual improvement as well, since best spectacle-corrected visual acuity rose for $95 \%$ of patients, a condition that justifies the very existence of this procedure, along with previously mentioned incisional techniques such as circular keratotomy and radial/ mini-radial keratotomy. Third, the long-term follow-up (7.3 years on average, six years minimum) supports the durability of these results, while it is also important to reiterate that no intra- or postoperative complications occurred.

More specifically, with reference to pachymetry, it is important to explain the increase in corneal thickness. Post-keratotomy fibrotic cellular responses produce thicker collagen fibrils over the incisions (along with corneal flattening), a process that is widely discussed in the literature as the review by Wilson et al. reports, ${ }^{20}$ without compromising the patient's vision due to their distance from the optical zone. This biomechanical process, known for decades, improves corneal pachymetry if the tissue's stability is maintained with appropriate surgery.

At the same time, it is understood that some notes of caution need to be discussed with the above.While other procedures are standardized in their execution, MARK is customized, since the number, length, position, and de pth of its mini-incisions vary depending on the cone's steepness and position, corneal thickness, and progression of Kc. Also, the inclusion and exclusion criteria that have been mentioned before need to be respected with care, with the aim of avoiding untoward complications deriving from inappropriate surgery, some which have been described in the literature. ${ }^{21}$ Further, as a corollary, the ophthalmic surgeon needs to be familiar with incisional surgery, both manual and laser, as well as with corneal sutures in the rare occasion of a microperforation. Lastly, it ought to be acknowledged that a randomized controlled trial would strengthen the internal validity of the data analyzed in this study.

\section{CONCLUSION}

Having outlined the strengths and the limitations of this technique, it is appropriate to contextualize it in the current treatment of Kc. We are witnessing an everincreasing precision in the diagnosis of keratoconus, which is also resulting in a growing number of patients who are diagnosed with one. The constant evolution of conservative procedures-CXL clearly among allas well as the refinement of DALK, are resulting in a lesser need for penetrating keratoplasty, all of which is reassuring news for keratoconic patients. Subsequently, one can conclude that the more options the ophthalmic surgeon has, the better it is for their treatment spectrum, which can, therefore, be adapted to the patient's specific needs.The long-term results analyzed by this study suggest that MARK should be considered as one of the conservative treatments for moderate to mild progressive keratoconus. In conclusion, however, we cannot stress enough the importance of prevention, as an early diagnosis of Kc would likely result in an earlystage CXL, which would in turn probably avoid the need for further surgery.

\section{REFERENCES}

1. Rabinowitz YS. Keratoconus. Surv Ophthalmol 1998;(42)4:297319.

2. Abdelaziz L, Barbara R. History of the development of the treatment of keratoconus. Int J Keratoconus and Ectatic Corneal Dis 2013;2(1):31-33.

3. Oh BL, Kim MK, Wee WR. Comparison of clinical outcomes of same-size grafting between deep anterior lamellar keratoplasty and penetrating keratoplasty for keratoconus. Korean J Ophthalmol 2013;27(5):322-30.

4. Wollensak G, Spoerl E, Seiler T. Riboflavin/ultraviolet-ainduced collagen crosslinking for the treatment of keratoconus. Am J Ophthalmol 2003;135(5):620-627.

5. Colin J, Cochener B, Savary G, et al. Correcting keratoconus with intracorneal rings. J of Cataract Refract Surg 2000;26(8):1117-1122.

6. Krumeich JH, Hirnschall N. Effect of Circular Keratotomy on Progression of Keratoconus. Int J Keratoconus and Ectatic Corneal Dis 2016;5(2):57-62.

7. Abbondanza M. Mini Cheratotomia Radiale Asimmetrica (Mini ARK) per la correzione chirurgica del cheratocono in fase iniziale, nell'ipermetropia e nelle miopie lievi. Esperienze 1997;12(1):21-24

8. Utine CA, Bayraktar S, Kaya V. Radial keratotomy for the optical rehabilitation of mild to moderate keratoconus: more than five years' experience. Eur J Ophthalmol 2006;16(3):376-384.

9. Fujimoto K, Osawa H, Moriyama T. Long-Term Stability of Minimally Invasive Radial Keratotomy for Mild to Moderate Keratoconus. Asia Pac J Ophthalmol 2017;6(5):407-411.

10. Alió JL, Claramonte PJ, Cáliz A. Corneal modeling of keratoconus by conductive keratoplasty. J Cataract Refract Surg 2005;31(1):190-197. 


\section{IJKECD}

11. Kanellopoulos AJ, Asimellis G. Keratoconus management: long-term stability of topography-guided normalization combined with high-fluence cxl stabilization (the Athens protocol). J Refract Surg 2014;30(2):88-93.

12. Kanellopoulos AJ, Pamel JP. Review of current indications for combined very high fluence collagen cross-linking and laser in situ keratomileusis surgery. Indian J Ophthalmol 2013;61(8):430-432.

13. El-Raggal TM. Sequential versus concurrent kerarings insertion and corneal collagen cross-linking for keratoconus. Br J Ophthalmol 2011;95(1):37-41.

14. Abbondanza M, Abbondanza G, De Felice V, et al. Longterm Results of Mini Asymmetric Radial Keratotomy and Corneal Cross-linking for the Treatment of Keratoconus. Kor J Ophthalmol 2019;33(2).

15. Kymionis GD, Kontadakis GA, Naoumidi TL. Conductive keratoplasty followed by collagen cross-linking with riboflavin-uv-a in patients with keratoconus. Cornea 2010;29(2):239-243.
16. Fadlallah A, Dirani A, El Rami H. Safety and visual outcome of vision toric icl implantation after corneal collagen cross-linking in keratoconus. J Refract Surg 2013;29(2): 84-89.

17. Abbondanza M, Abdolrahimzadeh B, Zuppardo M. Refractive Changes Following CXL. Cataract Refract Surg Today Eur 2009;4(7):33-38.

18. Lombardi M, Abbondanza M. Asymmetric radial keratotomy for the correction of keratoconus. J Refract Surg 1997;13(3):302-307.

19. Lindstrom RL. Minimally invasive radial keratotomy: MiniRK. J Cataract Refract Surg 1995;21(1):27-34.

20. Wilson SL, El Haj AJ, Yang Y. Control of Scar Tissue Formation in the Cornea: Strategies in Clinical and Corneal Tissue Engineering. J Funct Biomater 2012;3(3):642-687.

21. Lovisolo CF, Mularoni A, Calossi A, et al. Complications of refractive keratotomy. In: Alió JL, Azar DT, editors. Management of Complications in Refractive Surgery. Berlin: Springer; 2008. p. 197-224. 